\title{
Infecção natural por Platynosomum fastosum em felino doméstico no município de Alegre, Espírito Santo e sucesso no tratamento com praziquantel
}

\author{
[Natural infection by Platynosomum fastosum in domestic feline in Alegre, Espírito Santo, Brazil \\ and successful treatment with praziquantel]
}

\section{"Relato de Caso/Case Report"}

\author{
Nayara Camatta Campos ${ }^{1 *}$, Daniele Fassina de Siqueira ${ }^{2}$, Lívia Reisen Perin ${ }^{1}$, \\ Luanna Castro Oliveira ${ }^{3}$, Diefrey Ribeiro Campos ${ }^{4}$, Isabella Vilhena Freire Martins ${ }^{5}$
}

\author{
${ }^{1}$ Médica veterinária autônoma, Linhares-ES, Espírito Santo, Brasil. \\ ${ }^{2}$ Médica veterinária autônoma, Vitória-ES, Brasil. \\ ${ }^{3}$ Universidade Estadual do Norte Fluminense Darcy Ribeiro, Campos dos Goytacazes-RJ, Brasil. \\ ${ }^{4}$ Universidade Federal Rural do Rio de Janeiro, Seropédica-RJ, Brasil. \\ ${ }^{5}$ Universidade Federal do Espírito Santo, Alegre-ES, Brasil. \\ *Autor para correspondência/Corresponding author: E-mail: ncamattavet@ gmail.com
}

\begin{abstract}
Resumo
Platynosomum sp. é um trematódeo encontrado em áreas tropicais e subtropicais e acomete felinos domésticos, sendo a principal via de transmissão a ingestão das lagartixas, contendo as formas infectantes, as metacercárias e parasitando fígado, vesícula biliar e ductos biliares. Na maioria dos animais infectados cursa com a forma assintomática, porém, a severidade da doença está associada com a carga, o tempo e a resposta individual do animal parasitado. O exame coproparasitológico é fundamental para possível diagnóstico, pela visualização de ovos operculados. O estudo tem como objetivo relatar um caso de um felino doméstico naturalmente infectado por Platynosomum fastosum no município de Alegre, Espírito Santo. A felina possuía acesso à rua com hábito de caçar e foi submetida a exame coproparasitológico para estudo epidemiológico de verminoses de gatos do município de Alegre-ES. No exame foi encontrado ovos de P. fastosum por meio da técnica parasitológica de centrífugo-flutuação. Não foram vistas alterações clínicas e no exame ultrassonográfico. A partir disso, foi tratada com praziquantel na dose de $20 \mathrm{mg} / \mathrm{kg}$ continuado por 3 dias. O exame coproparasitológico foi repetido uma semana e 10 meses após o tratamento obtendo-se resultados negativos evidenciando o sucesso da terapêutica. Dessa forma, os dados presentes nesse relato buscam atentar para casos de platinossomíases e introduzir os exames coproparasitológicos na rotina.
\end{abstract}

Palavras-chave: colangite parasitária; platinosomíase; gato.

\begin{abstract}
Platynosomum sp. is a trematode found in tropical and subtropical areas that affects domestic felines, the main route of transmission being the ingestion of lizards containing the infective forms, the metacercariae, and parasitizing the liver, gall bladder and bile ducts. In most infected animals, the disease is asymptomatic, but the severity of the disease is associated with the burden, time, and individual response of the parasitized animal. The coproparasitological examination is fundamental for possible diagnosis, which is based on the visualization of the eggs. The study aims to report a case of a domestic feline naturally infected by Platynosomum fastosum in the city of Alegre, Espírito Santo. The feline had access to the street with a habit of hunting and underwent a coproparasitological examination for the epidemiological study of cats in the city of Alegre-ES. In the examination, eggs of $P$. fastosum were found by means of the centrifugal-flotation parasitological technique. No clinical changes or on ultrasound examination were seen. Thereafter, treatment was instituted with praziquantel at a dose of $20 \mathrm{mg} / \mathrm{kg}$ for 3 days. The coproparasitological examination was repeated one week and 10 months after the treatment, obtaining negative results and evidencing the success of the therapy. In this way, the data present in this report seeks to draw attention to cases of platinosomiasis and to introduce routine coproparasitological exams.
\end{abstract}

Keywords: parasitic cholangitis; platinosomiasis; cat. 


\section{Introdução}

A platinosomíase, popularmente conhecida como envenenamento por lagartixa, é uma doença parasitária das vias biliares, comum em regiões tropicais e subtropicais. Geralmente habita o fígado, vesícula biliar, ductos biliares e menos comumente, o intestino delgado e pâncreas dos felinos domésticos (Norsworthy, 2011).

Seu ciclo exige a presença de dois a três hospedeiros intermediários que podem ser isópodos e moluscos terrestres, considerados os primeiros. As lagartixas e sapos sendo os segundos hospedeiros intermediários. A principal via de transmissão nos gatos é pela ingestão das lagartixas, contendo as formas infectantes, as metacercárias (Newell et al., 2001; Basu e Charles, 2014; Pinto et al., 2014).

O estilo de vida do gato influencia na prevalência deste parasito, sendo que em animais de vida livre a ocorrência é cerca de 15 a $85 \%$, em animais confinados é de $7,1 \%$ e nos semiconfinados o valor é de 28,6\% (Salomão et al., 2005). Fêmeas têm $95 \%$ mais probabilidade de se infectarem, devido à necessidade que elas têm de caçar para alimentarem seus filhotes (Foley, 1994; Rodriguez-Vivas et al., 2004).

O diagnóstico definitivo é realizado pela detecção de ovos castanhos e operculados nas fezes (Foley, 1994). A ultrassonografia pode ser utilizada no diagnóstico diferencial entre obstrução biliar e doença hepatobiliar, podendo-se observar dilatação dos ductos biliares e distensão da vesícula biliar, com bile ecogênica. Também pode ser observado hepatomegalia e distensão da árvore biliar hepática (Foley, 1994; Tams, 1994; Javinsky, 2011).

O praziquantel é o fármaco de escolha para o tratamento da platinosomíase. Relata-se dose de praziquantel de 10 a $20 \mathrm{mg} / \mathrm{kg}$, por via subcutânea ou oral, um comprimido ao dia, de 3 a 5 dias consecutivos, repetindo a mesma posologia após 12 semanas de intervalo, pois os ovos do parasito podem estar presentes nas fezes até 9 semanas após o tratamento (Zanutto et al., 2012).

Casos relatados de platinossomíase no Brasil são escassos principalmente pelos sinais inespecíficos da doença como icterícia, anorexia, letargia, perda de peso, vômito e desidratação desencadeando quadros de doença hepática como a colangiohepatite (Soldan e Marques, 2011). Dessa forma, o diagnóstico é subestimado, não sendo realizados exames coproparasitológicos de rotina e muitas vezes o parasito é encontrado como achado pós-morte, como relatado por Gava et al. (2015), no Espírito Santo, em que um felino fêmea e adulta teve morte súbita, encontrando-se, à necropsia o parasito no fígado com presença de colangiohepatite.

Devido à grande importância de se enfatizar a infecção pelo parasito, de suas lesões nos felinos, diagnóstico e tratamento o estudo teve como objetivo relatar um caso de platinosomíase felina no município de Alegre, Espírito Santo, com sucesso no tratamento.

\section{Descrição do Caso}

Uma fêmea felina, sem raça definida, seis meses de idade, foi adotada, sem relato de vacinação ou vermifugação com acesso à rua e hábito de caçar. $\mathrm{O}$ animal foi submetido ao exame coproparasitológico de rotina realizado no Laboratório de Parasitologia Animal do Hospital Veterinário da Universidade Federal do Espírito Santo (HOVET - CCA UFES).

O exame coproparasitológico foi realizado utilizando a técnica de sedimentação em formalinaéter (Ritchie, 1948) e a técnica de centrífugoflutuação (Sloss et al., 1999). Porém, o mesmo somente foi positivo para infecção por $P$. fastosum na técnica de centrífugo-flutuação, não demonstrando uma alta carga parasitária.

Após o diagnóstico o gato foi submetido a uma avaliação clínica, na qual nenhuma alteração foi registrada. Também foi realizado o exame ultrassonográfico, que não demonstrou nenhuma alteração hepática ou de vesícula biliar.

O felino foi tratado segundo recomendado por Foley (1994), que preconizou a utilização de praziquantel na dose de $20 \mathrm{mg} / \mathrm{kg}$ por 3 dias consecutivos e repetição da posologia após 12 semanas. $\mathrm{O}$ tratamento foi realizado, porém não houve repetição da dose após 12 semanas do primeiro tratamento. Uma semana e 10 meses após o tratamento foram realizados novos exames coproparasitológicos utilizando as mesmas técnicas com a coleta de amostras por 3 dias seguidos, não demonstrando nenhuma infecção parasitária por $P$. fastosum pós-tratamento.

\section{Discussão}

Durante o exame clínico do animal não se observou nenhuma alteração o que é compatível com encontrado por outros autores em que as manifestações clínicas associadas ao parasitismo 
por $P$. fastosum variam conforme a severidade, que está associada com a carga, o tempo e a resposta individual do animal parasitado e o tempo de infecção (Bielsa e Greiner, 1985; Foley, 1994; Salomão et al., 2005).

Felinos não domiciliados ou com acesso à rua possuem maior prevalência de infecção por $P$. fastosum quando comparado com animais domiciliados que não possuem acesso à rua (Salomão et al., 2005). O animal em questão, era semi domiciliado, possuía acesso à rua e hábito de caçar. A maior predisposição se dá, sobretudo, quando há histórico de ingestão de pequenos lagartos (Foley, 1994; Watson, 2014).

O gato possuía seis meses de idade Salomão et al. (2005) descreveram que filhotes podem ser infectados a partir de presas fornecidas pela mãe. Além disso, fêmeas que caçam possuem $95 \%$ maior probabilidade de se infectarem, possivelmente por terem que alimentar os filhotes (Foley, 1994)

O diagnóstico foi realizado por meio do exame coproparasitológico, sendo esta, a forma de diagnóstico definitivo, quando os ovos são encontrados. Normalmente, um pequeno número de ovos está presente nas fezes e a ovoposição dos parasitos é intermitente, tornando esse método de diagnóstico pouco sensível, o que pode resultar em falsos negativos. Desta forma, é recomendado o exame fecal seriado. Os ovos podem ser encontrados na técnica de centrifugo flutuação, porém mais recomendada, por ser considerada a mais sensível, é a de sedimentação em formalinaéter (Taylor e Perri, 1977; Norsworthy, 2011).

Apesar de alguns autores afirmarem que a técnica de sedimentação em formalina-éter é a técnica de eleição para a platinossomíase por possui maior sensibilidade (Norsworthy, 2011), alguns trabalhos demonstraram que a técnica de centrífugo-flutuação também pode detectar ovos desse trematódeo (Rocha et al., 2014), como observado no presente estudo.

O exame ultrassonográfico possibilita uma identificação precisa do fígado quanto ao tamanho hepático, seja em estado normal ou alterado, demonstrando melhor diferenciação dos limites dos órgãos (Daniel et al., 2012; Azevedo et al., 2013; Montserin et al., 2013). A infecção por $P$. fastosum pode influenciar o aspecto da parede da vesícula biliar no exame ultrassonográfico de felinos, aparentando uma hiperecogenicidade na vesícula, que pode ser explicada pela inflamação causada pelo trematódeo (Salomão et al., 2005).
Animais parasitados também podem apresentar hepatomegalia, contornos hepáticos irregulares e parede vesicular aumentada (Azevedo et al., 2013). Essas alterações normalmente são observas em animais com infecções crônicas ou alta carga parasitária (Salomão et al., 2005; Soldan e Marques, 2011).

O exame ultrassonográfico do felino em questão não mostrou nenhuma alteração de vesícula biliar, o único achado foi a vesícula bilobada o que é comum na espécie felina. Este resultado é compatível com Salomão et al. (2005) que afirmaram que alterações decorrentes da platinosomíase começam a aparecer no exame ultrassonográfico nos estágios avançados da doença.

O tratamento utilizado foi praziquantel, fármaco de escolha para a platinosomíase (Norsworthy, 2011; Zanutto et al., 2012). Foley (1994) e Norsworthy (2011) comentaram da utilização de $20 \mathrm{mg} / \mathrm{kg}$ por três dias consecutivos, via oral. Os autores também sugeriram que se repita a posologia após 12 semanas de intervalo, pois os ovos do parasito podem estar presentes nas fezes até 9 semanas após o tratamento, devido à sobrevivência do trematódeo no sistema biliar ou retidos na árvore biliar. A repetição da dose não foi realizada, porém o exame do animal uma semana e 10 meses após o tratamento não mostrou nenhum parasitismo, mesmo com o animal mantendo seus hábitos de caçar e tendo acesso à rua. Neste caso o praziquantel foi efetivo na eliminação do parasitismo.

Como prevenção para animais que vivem em área endêmica, preconiza-se a utilização de praziquantel na dose de $20 \mathrm{mg} / \mathrm{kg}$, via oral, a cada 3 meses, sendo importante limitar o acesso do animal à rua e a hospedeiros intermediários, assim como exames coproparasitológicos periódicos (Norsworthy, 2011; Zanutto et al., 2012).

No Brasil existem poucos estudos sobre a platinosomíase, mesmo com o crescente número de casos em vários estados do País. A ocorrência do $P$. fastosum no município de Alegre, Espírito Santo, demonstra a necessidade de investigar a sua casuística e avaliar a importância da doença no estado do Espírito Santo.

\section{Conclusão}

O tratamento com o praziquantel na dose de $20 \mathrm{mg} / \mathrm{kg}$ durante 3 dias mostra-se eficaz na eliminação do parasitismo por $P$. fastosum. 
Embora o caso relatado seja um achado de projeto de extensão, e não um caso clínico, é importante que médicos veterinários da região de Alegre, Espírito Santo, se atentem para casos de doenças hepáticas, podendo ser a infecção por $P$. fastosum diagnóstico diferencial.

\section{Conflito de Interesse}

Os autores declaram não existir conflito de interesse.

\section{Comitê de Ética}

O trabalho foi aprovado pelo comitê de ética na utilização de animais da Universidade Federal do Espírito Santo, sob o número 031/2013.

\section{Referências}

Azevedo, F.D.; Veiga, C.C.P.; Scott, F. B.; Azevedo, T. R. C.; Souza, B. G.; Vulcano, L. C. Avaliação radiográfica e ultrassonográfica do fígado e da vesícula biliar em gatos domésticos (Felis catus domesticus) parasitados por Platynosomum illiciens (Braun, 1901) Kossak, 1910. Revista Brasileira de Medicina Veterinária, 35(2): 283-288, 2013.

Basu, A.K.; Charles, R.A. A review of cat liver fluke Platynosomum fastosum Kossack, 1910 (Trematoda: Dicrocoeliidae). Veterinary Parasitology, 200 (1-2): 1-7, 2014.

Bielsa, L.M.; Greiner, E.C. Liver Fluke (Platynosomum concinnum) in cats. Journal of the American Animal Hospital Association, 21:269-274, 1985.

Daniel, A.G.T.; Diaz, R.F.; Camignatto, L.O.; Kage, N.K.; Pellegrino, A.; Cogliati, B. Polycystic Liver Disease Associate with Platynosomum fastosum Infection in Cat. Brazilian Journal of Veterinary Pathology, 5(3): 137-141, 2012.

Foley, R.H. Platynosomum concinnum infection in cats. Compendium on Continuing Education for the Practising Veterinarian, 16(10): 1271-1277, 1994.

Gava, M.G.; Hiura, E.; Lopes, A.D.C.; Vieira, F.T.; Flecher, M.C.; Fonseca, L.A.; Soares, F.E.F.; Giuberti, T.Z.; Leite, F.L.G.; Lenz, D.; Rassele, A.C.; Paz, J.S.; Alves, A.; Braga, F.R. Platynosomum fastosum in an asymptomatic cat in the state of Espírito Santo: first report. Revista de Patologia Tropical, 44(4): 496-502, 2015.
Javinsky, E. Gastrointestinal parasites. In: Little, S. The cat: clinical medicine and management. Saint Louis: Elsevier, 2011. p. 1424.

Montserin, S.A.S., Munoz, K., Seebaransingh, R., Basu, A.K. Clinical case: Platynosomum fastosum Kossack, 1910 infection in a cat: first reported case in Trinidad and Tobago. Revue de Médecine Vétérinaire, 164: 9-12, 2013.

Newell, S.M.; Graham, J.P.; Roberts, G.D.; Ginn, P.E.; Greiner, E.C.; Cardwell, A.; Mauragis, D.; Knutsen, C.; Harrison, J.M.; Martin, F.G. Quantitative hepatobiliary scintigraphy in normal cats and in cats with experimental cholangiohepatitis. Veterinary Radiology \& Ultrasound, 42:70-76, 2001.

Norsworthy, G.D. Flukes: Liver, biliary and pancreatic. In: Norsworthy, G.D., Crystal, M.A., Grace, S.F., Tilley, L.P. The feline patient. Blackwell, 2011. p.193-194.

Pinto, H.A.; Mati, V.L.; De Melo, A.L. New insights into the life cycle of Platynosomum (Trematoda: Dicrocoeliidae). Parasitology Research, 113(7): 2701-2707, 2014.

Ritchie, L.S. An ether sedimentation technique for routine stool examination. Bulletin of the United States Army Medical Department, 8: 326, 1948.

Rocha, N.O.; Portela, R.W.; Camargo, S.S.; Souza, W.R.; Carvalho, G.C.; Bahiense, T.C. Comparison of two coproparasitological techniques for the detection of Platynosomum sp. infection in cats. Veterinary Parasitology, 29: 392-395, 2014.

Rodriguez-Vivas, R.I, Williams, J.J.; QuijanoNovelo, A.G.; Bolio, G.M., Torres-Acosta, J.F. Prevalence, abundance and risk factors of liver fluke (Platynosomum concinnum) infection in cats in Mexico. The Veterinary Record, 154:693- 694, 2004.

Salomão, M.; Souza-Dantas, L.M.; Mendes-DeAlmeida, F.; Branco, A. S.; Bastos, O.P.M.; Sterman, F.; Labarthe, N. Ultrasonography in Hepatobiliary Evaluation of Domestic Cats (Felis catus, L., 1758) Infected by Platynosomum Looss, 1907. International Journal of Applied Research in Veterinary Medicine, 3:271-279, 2005.

Sloss, M. W.; Zajac, A. M.; Kemp, R. L. Parasitologia clínica veterinária. São Paulo: Manole, 1999. p.198. 
Soldan, M.H.; Marques, S.M.T. Platinosomose: abordagem na clínica felina. Revista da FZVA, 18(1):46-67, 2011.

Tams, T.R. Hepatobiliary parasites In: Sherding, R.G. The cat: diseases and clinical management, $2^{\mathrm{a}}$ ed. Philadelphia: Saunders. 1994. p. 607.

Taylor, D.; Perri, S.F. Experimental infection of cats with the liver fluke Platynosomum concinnum. American Journal of Veterinary Research, 38(1):51-55, 1977.
Watson, P.J. Clinical manifestations of hepatobiliary disease. In: Nelson, R.W.; Couto, C.G. Small animal internal medicine, 2014. p. 501-511.

Zanutto, M.S.; Almeida, M.A.O.; Junquilho, A.B.; Silva, M.S.A.; Silveira, R. X.; Fatal, P.L. Uso do Endal Gatos no tratamento da platinossomíase felina. A Hora Veterinária, 185: 12-16, 2012. 